\title{
Communication management in global software development projects
}

\begin{abstract}
Many organizations that have the global software development (GSD) projects use communication technologies to connect their virtual teams. However, the virtual team faces various challenges and issues in the process of the GSD. One of the significant challenges is obtaining an efficient communication among team members. This study focuses on the communication factors among the virtual teams highlighted in the literature. The communication factors are related to the temporal distances, geographical distances, sociocultural, access to training, technological problems that hindering communications, the communication within the status of development process, personal communication skills and language differences. The objective of this study is to examine the communication factors and identify the related issues which are commonly occurring between the virtual teams in the global software development. A survey was conducted in different sectors. The findings indicate that some of important points related to the communication factors contribute to the success of the GSD.
\end{abstract}

Keyword: Project management; Team communication; Communication management; Global software development 